\title{
Methamphetamine Induces Dopamine Release in the Nucleus Accumbens Through a Sigma Receptor-Mediated Pathway
}

\author{
David M Hedges', J Daniel Obray², Jordan T Yorgason², Eun Young Jang ${ }^{2}$, Vajira K Weerasekara', \\ Joachim D Uys ${ }^{3}$, Frederick P Bellinger ${ }^{4}$ and Scott C Steffensen ${ }^{*, 2}$ \\ 'Department of Chemistry and Biochemistry, Brigham Young University, Provo, Utah, USA; ${ }^{2}$ Department of Psychology and Neuroscience, Brigham \\ Young University, Provo, UT, USA; ${ }^{3}$ Department of Cell and Molecular Pharmacology, Medical University of South Carolina, Charleston, SC, USA; \\ ${ }^{4}$ Department of Cell and Molecular Biology, University of Hawaii at Manoa, Honolulu, HI, USA
}

\begin{abstract}
Methamphetamine (METH) is a drug with a high addictive potential that is widely abused across the world. Although it is known that METH dysregulates both dopamine transmission and dopamine reuptake, the specific mechanism of action remains obscure. One promising target of $\mathrm{METH}$ is the sigma receptor, a chaperone protein located on the membrane of the endoplasmic reticulum. Using fastscan cyclic voltammetry, we show that METH-enhancement of evoked dopamine release and basal efflux is dependent on sigma receptor activation. METH-induced activation of sigma receptors results in oxidation of a cysteine residue on VMAT2, which decreases transporter function. Unilateral injections of the sigma receptor antagonist BD-1063 prior to METH administration increased dopamine-related ipsilateral circling behavior, indicating the involvement of sigma receptors. These findings suggest that interactions between METH and the sigma receptor lead to oxidative species (most likely superoxide) that in turn oxidize VMAT2. Altogether, these findings show that the sigma receptor has a key role in METH dysregulation of dopamine release and dopamine-related behaviors.

Neuropsychopharmacology (2018) 43, I405-1414; doi:I0.1038/npp.2017.291; published online 24 January 2018
\end{abstract}

\section{INTRODUCTION}

Methamphetamine (METH) is an incredibly addictive psychostimulant with abuse presenting a growing problem throughout the world. Understanding the mechanisms underlying METH's reinforcing properties will help identify pharmaceutical targets for more effective treatment options for affected individuals as well as prevention. The mesolimbic dopamine (DA) system, consisting of DAergic neurons projecting from the midbrain to target areas in the striatum and nucleus accumbens (NAc), has been implicated in reward and motivation for drugs of abuse including METH (Robinson and Berridge, 1993; Sulzer et al, 2005).

One high-affinity target of METH is the sigma-1 receptor $(\sigma 1 \mathrm{R})$. The $\sigma 1 \mathrm{R}$ is a ligand-gated chaperone protein that, upon activation, translocates to various cellular compartments of the endoplasmic reticulum (ER) where it is thought to affect protein folding, ion-channel, and G-protein coupled-receptor (GPCR) function, resulting in the formation of mitochondrial-sourced reactive oxygen species (ROS) (Fukunaga et al, 2015; Katz et al, 2011; Nguyen et al, 2014; Su et al, 2010). Blocking $\sigma 1$ Rs prevents both METH-induced

\footnotetext{
*Correspondence: Dr SC Steffensen, Department of Psychology and Neuroscience, Brigham Young University, 1050 SWKT, Provo UT 84602, USA, Tel: +|-80|-422-9499, Fax: +|-80|-422-0602, E-mail: scott_steffensen@byu.edu

Received 15 August 2017; revised 14 November 2017; accepted 16 November 2017; accepted article preview online 29 November 2017
}

ROS generation and DA release in cultured NG108 cells (Kaushal et al, 2012). Thus, METH's actions at $\sigma 1$ Rs may be a possible underlying mechanism behind the formation of METH-generated ROS and subsequent DA release in vivo. Moreover, many $\sigma 1 \mathrm{R}$ agonists substitute for both METH and cocaine in self-administration paradigms (Hiranita et al, 2013; Katz et al, 2011), further implicating this protein and its downstream pathway(s) in the reinforcing effects of METH.

METH is a powerful modulator of the DA system and has a myriad of effects that influence DA release. It disrupts DA reuptake and packaging systems, likely through direct effects on the DA transporter (DAT) (McFadden et al, 2015; Siciliano et al, 2014) and the vesicular monoamine transporter 2 (VMAT2) (Chu et al, 2008; Fleckenstein et al, 2009; Siciliano et al, 2014). Through this process, METH is thought to increase cytoplasmic levels of DA that overwhelm the active transport of the DAT, leading to reverse transport of DA (cytoplasm to synaptic cleft) and eventual depletion of DA stores (Eshleman et al, 1994; Han and Gu, 2006; Jones et al, 1998a; 1998b; Rothman and Baumann, 2003; Sitte et al, 1998). METH may also influence DA release through its prooxidant actions and downstream effects of resulting ROS. For instance, a dose of METH that leads to general DA depletion $(10 \mathrm{mg} / \mathrm{kg})$ also induces formation of the freeradical peroxynitrite (Imam et al, 2001b), a reactive nitrogen species formed by the interaction between superoxide and nitric oxide. Furthermore, overexpressing a copper-zinc superoxide dismutase protects against both peroxynitrite 
formation as well as METH-induced DA depletion (Imam et al, 2001b), indicating that superoxide is involved in METH's DA depleting effects. Importantly, we have previously shown that ROS scavengers have also been shown to be effective at attenuating METH's DA-enhancing effects (Jang et al, 2017). In this report, we examine the role and downstream mechanism of $\sigma 1 \mathrm{Rs}$ in METH's excitatory effects on DA release and METH-induced circling behavior.

\section{MATERIALS AND METHODS}

\section{Animal Subjects}

Male C57BL/6 mice (PND $30-120$ ) and Wistar rats (PND $80-120)$ were bred and cared for in accordance with the National Institutes of Health Guide for the Care and Use of Laboratory Animals. At weaning (PND 21), animals were housed on a reverse light/dark cycle (lights on from 2000 hours to 0800 hours) in groups of $2-5$ per cage and given ad libitum access to food and water. Experimental protocols were approved by the Brigham Young University Institutional Animal Care and Use Committee according to $\mathrm{NIH}$ guidelines.

\section{Brain Slice Preparation}

Coronal brain slices were obtained as previously described (Steffensen et al, 2008). Animals were anesthetized with isoflurane (5\%), decapitated, and brains were dissected and sectioned into $400 \mu \mathrm{m}$ slices in ice-cold cutting solution consisting of (in $\mathrm{mM}$ ): 220 sucrose, $3 \mathrm{KCl}, 1.25 \mathrm{NaH}_{2} \mathrm{PO}_{4}, 25$ $\mathrm{NaH}_{2} \mathrm{CO}_{3}, 12 \mathrm{MgSO}_{4}, 10$ glucose, and $0.2 \mathrm{CaCl}_{2}$. Slices were then transferred to room temperature artificial cerebrospinal fluid (ACSF) consisting of (in mM): $124 \mathrm{NaCl}, 2 \mathrm{KCl}, 1.25$ $\mathrm{NaH}_{2} \mathrm{PO}_{4}, 24 \mathrm{NaHCO}_{3}, 12$ glucose, $1.2 \mathrm{MgSO}_{4}, 2 \mathrm{CaCl}_{2}, \mathrm{pH}$ 7.3 , which was bubbled with $95 \% \mathrm{O}_{2} / 5 \% \mathrm{CO}_{2}$. Slices were then transferred to a recording chamber with continuous ACSF flow $(2.0 \mathrm{ml} / \mathrm{min})$ maintained at $34-36^{\circ} \mathrm{C}$. Unless otherwise specified, all drugs were purchased from Sigma Aldrich (St. Louis, Missouri, USA). For slice preparation experiments, METH, lidocaine, GBR-12909 (Cayman Chemical, Ann Arbor, MI, USA), BD-1063 (Cayman Chemical), CPA (Cayman Chemical), TEMPOL, and GSH were dissolved in stock solutions and then diluted into ACSF at specified concentrations.

\section{Fast-Scan Cyclic Voltammetry Recordings}

Carbon fiber electrodes (CFEs) were positioned at an angle, $\sim 75 \mu \mathrm{m}$ below the surface of the slice in the NAc. DA release was evoked every $2 \mathrm{~min}$ by biphasic electrical stimulation ( $4 \mathrm{~ms}$ pulses, 10 pulse, $350 \mu \mathrm{A}, 20 \mathrm{~Hz}$ ) from an ACSF filled micropipette $(5-10 \mu \mathrm{m}$ tip diameter), placed $100-200 \mu \mathrm{m}$ from the CFE. The CFE potential was linearly scanned from -0.4 to $1.2 \mathrm{~V}$ and back to $-0.4 \mathrm{~V}$ vs $\mathrm{Ag} / \mathrm{AgCl}$ (scan rate $=400 \mathrm{~V} / \mathrm{s})$. Cyclic voltammograms were recorded every $100 \mathrm{msec}(10 \mathrm{~Hz})$ with ChemClamp potentiometers (Dagan Corporation, Minneapolis, MN, USA). Recordings were performed and analyzed using Demon Voltammetry software (Yorgason et al, 2011).

For DA efflux experiments, stimulated DA release was detected first to verify CFE placement. When electrically evoked DA release did not vary by more than $5 \%$ for five successive collections, the electrical stimulation was turned off and voltammograms were recorded at $0.5 \mathrm{~Hz}$ for $3 \mathrm{~h}$. Unless otherwise specified, all other recordings were performed with the pharmacological agent added at $30 \mathrm{~min}$ and METH at $60 \mathrm{~min}$ from the start of the recording. DA release and efflux is expressed as a change in $\mu \mathrm{M} \mathrm{DA}$, not absolute extracellular DA concentration, due to the background subtracted nature of voltammetric recordings.

\section{VMAT2 Co-Immunoprecipitation Assay and Immunoblot}

Aged-matched subjects were divided into three groups that were then double-injected intraperitoneally with saline/ saline, saline/METH $(10 \mathrm{mg} / \mathrm{kg})$, and BD-1063 (30 mg/kg)/ METH $(10 \mathrm{mg} / \mathrm{kg})$, respectively. The injections were $\sim 10$ min apart. NAc tissue was immediately harvested and rapidly chilled $30 \mathrm{~min}$ after the second injection. The tissue samples were lysed in ice-cold TNTE buffer (in mM: 50 Tris $\mathrm{pH} 7.4,150 \mathrm{NaCl}$, and 1 EDTA in addition to $0.5 \%$ Triton $\mathrm{X}-100$ ) for $10 \mathrm{~min}$ with a rotation at $4{ }^{\circ} \mathrm{C}$. Tissue lysates were triterated 10 times using a 23 gauge needle and cleared by centrifugation at $14800 \mathrm{~g}$ for $10 \mathrm{~min}$. Anti-VMAT2 antibody (EMD Millipore, Billerica, MA, USA) was coupled to magnetic dynabeads (ThermoFisher, Waltham, MA, USA) and immunoprecipitated VMAT2 from tissue lysates according to the manufacturer's instructions. Immunoprecipitated VMAT2 was run on a $10 \%$ SDS-PAGE and immunoblotted for anti-GSH (Virogen, Watertown, MA, USA) and antiVMAT2 antibodies. After immunoblotting, samples were analyzed/quantified using fluorescent secondary antibodies and the Li-Cor Odyssey imaging system.

\section{Circling Behavior Assay}

Adult male Wistar rats $(n=10,>400 \mathrm{~g}$ at the start of the experiment) were implanted with a guide cannula (MD-2250, Basi Instruments, West Lafayette, IN, USA) in the $\mathrm{NAc}(+1.2$ to $+1.7 \mathrm{AP},-0.8$ to $-1.6 \mathrm{ML},+6.0$ to $+7.0 \mathrm{DV})$, which was fixed in place using dental cement. Subjects were maintained under isoflurane anesthesia $(1.5-2.0 \%)$ throughout the surgical procedure and allowed a minimum of 1 week of recovery time. Circling experiments were performed in a $16 \times 16 \times 16$ inch plexiglass compartment. Rats were briefly anesthetized using isoflurane (4.0\%) during injections to mitigate injection stress. On test days, rats were injected intraperitoneally with either $5 \mathrm{mg} / \mathrm{kg} \mathrm{METH}$ dissolved at $5 \mathrm{mg} / \mathrm{ml}$ in $0.9 \% \mathrm{NaCl}$ saline solution or $1 \mathrm{ml} / \mathrm{kg} 0.9 \% \mathrm{NaCl}$ saline solution. This injection was followed $5 \mathrm{~min}$ later by either a unilateral $0.5 \mu \mathrm{l}$ injection of $5 \mu \mathrm{g}$ BD-1063 dissolved in ACSF or a $0.5 \mu \mathrm{l}$ unilateral injection of ACSF into the NAc. Microinjections were accomplished using a microinjection pump (A-99, Razel Scientific Instruments, Fairfax, VT, USA) and a $25 \mu \mathrm{l}$ glass syringe (Hamilton Robotics, Reno, NV, USA) connected by silastic tubing to an injection needle that extended $0.5-1.0 \mathrm{~mm}$ past the end of the guide cannula. The injection occurred over $30 \mathrm{~s}$, with the needle left in place for an additional $30 \mathrm{~s}$ prior to removal. After injections, one hour of behavior in the circling chamber was recorded using a camera mounted on the ceiling above the apparatus connected to a Windows 7 PC running Pinnacle 
Studio 16 (Corel, Menlo Park, CA, USA). Injection order was counterbalanced across rats to avoid order effects. At the end of the experiment, rats were injected with $0.5 \mu \mathrm{l}$ of $100 \mathrm{mM}$ pontamine sky blue (Avocado Research Chemicals Limited, Lancashire, UK) dissolved in distilled water. Rats were then killed and their brains were removed for sectioning using a vibratome. Sectioned brain slices were examined to determine microinjection location. Two rats were removed from the experiment, one due to chronic distress and a second due to improper injection location. In the distressed rat an autopsy revealed that the lower portion of the guide cannula had disconnected from the implant resulting in an extensive striatal lesion. Two additional rats did not complete the
saline+BD-1063 experimental condition despite completing all other conditions.

\section{Statistical Analyses}

Immediately following METH administration, the amplitude of electrically evoked DA release increased, but dropped after several collection sweeps. For the sake of directly comparing against DA efflux, we averaged the maximal three peak DA amplitude measurements. For statistical measure of DA efflux, the results were determined from maximum oxidation peak values for evoked DA experiments. As the DA efflux evoked by METH generally decreased after 30-60 min, the area under the curve (AUC) between the initial DA increase and the return to baseline was calculated using Igor Pro
Evoked DA Release

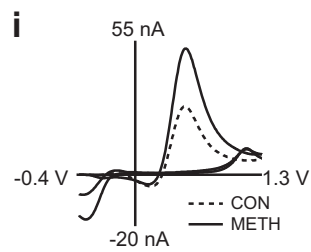

ii

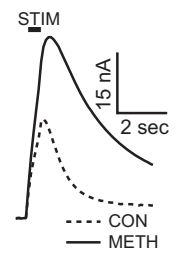

b
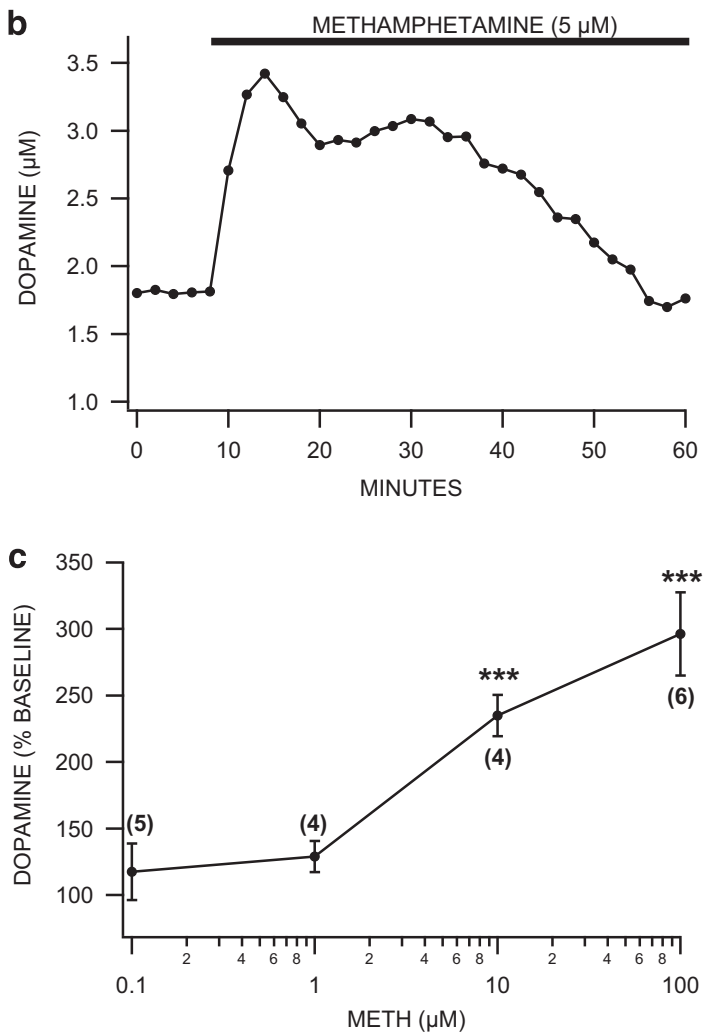

d

\section{DA Efflux}

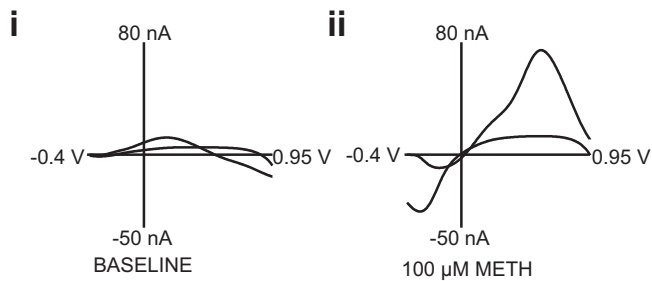

e

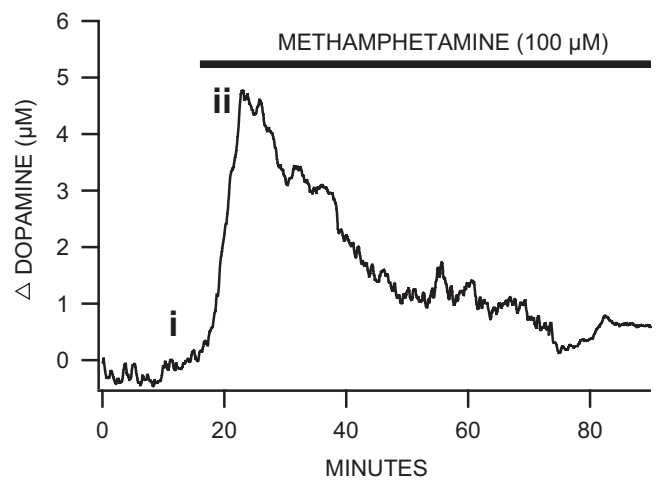

f

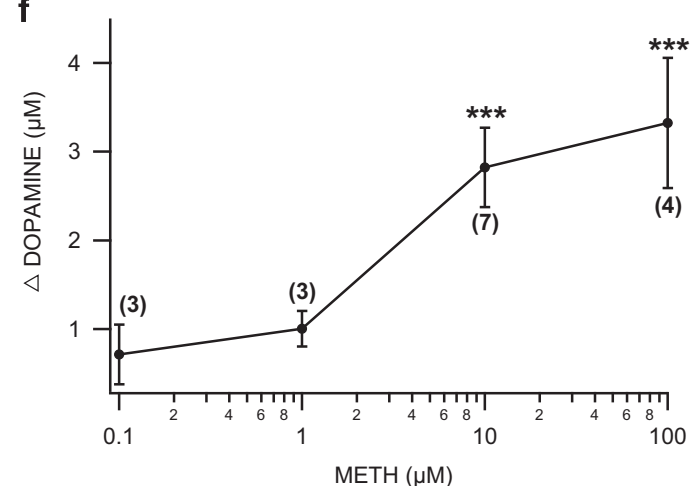

Figure I Methamphetamine markedly enhanced electrically evoked dopamine release and dopamine efflux in the nucleus accumbens core ex vivo. (a) Representative superimposed cyclic voltammograms (Ai) and current vs time (Aii) evoked DA release plots recorded in the core of the NAc slice preparation and evoked by local stimulation (STIM) in the slice preparation before and after superfusion of METH. (b) In this representative example, METH markedly enhanced evoked DA release at $5 \mu M$ (the three peak values were taken for analysis) with recovery in approximately I h $(n=I I)$. (c) Dose-response curve showing that METH significantly enhanced evoked DA release with an estimated $\mathrm{EC}_{50}$ of $5 \mu \mathrm{M}$. (d) Representative cyclic voltammograms of spontaneous DA efflux before (BASELINE; left) and after 100 HM METH (right). (e) In this representative example, METH markedly enhanced DA efflux with recovery in about I h. Although the $\mathrm{EC}_{50}$ was estimated to be $5 \mu \mathrm{M}$, this representative trace at $100 \mu \mathrm{M}$ METH was chosen to show a cleaner voltammogram to confirm that this is indeed causing DA release. ( $f$ ) Dose-response curve showing that METH significantly enhanced DA efflux (AUC) with an estimated EC 50 of $5 \mu M$. Values in parentheses indicate $n$ values. Asterisks (****) represent significance level $p<0.00$ I. DA, dopamine; METH, Methamphetamine; NAc, nucleus accumbens. 

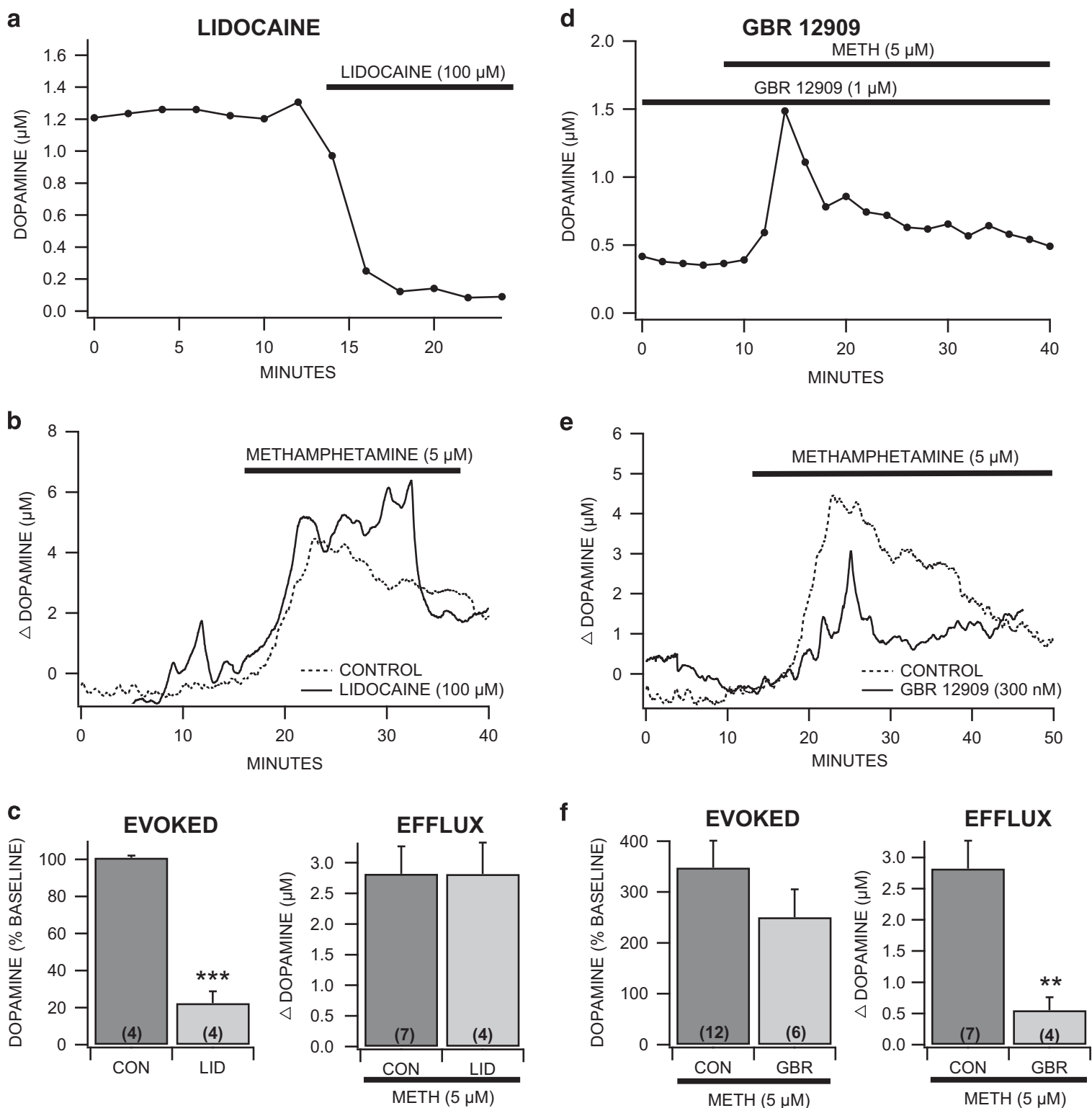

Figure 2 Mechanistic pharmacology of METH-enhancement of dopamine release. (a, c) Lidocaine (I00 $\mu M)$ abolished evoked DA release in the core of the NAc slice preparation $(n=5)$. (b, c) METH-induced DA efflux was unaffected by lidocaine. ( $d$, f) Methamphetamine enhancement of evoked DA release was unaffected by the selective DAT inhibitor GBR-12909 (I HM) in the slice $(n=6)$. (e, f) GBR-I2909 reduced METH-induced DA efflux. Values in parentheses indicate $n$ values. Asterisks (**, ****) represent significance levels $p<0.01$ and $p<0.00$ I, respectively. DA, dopamine; DAT, DA transporter; METH, Methamphetamine.

(WaveMetrics, Lake Oswego, OR, USA). The AUC was then divided by the $\Delta t$ of the DA efflux for the averaged data plots. This standardized experiments for average DA release per second. These values were then grand-averaged across animals. Values were expressed as means \pm SEM for cumulated data.

Statistics were performed using IBM SPSS Statistics 21 (Armonk, NY, USA) or STATA 14.2 (College Station, TX, USA). A two-tailed Student's $t$ test was used for comparisons with only two variables. To determine significance between more than two variables, a one-way analysis of variance (ANOVA) was used with Tukey's post hoc analysis. Circling and locomotor activity was analyzed using repeated measures ANOVAs with a Greenhouse-Geisser correction for sphericity. Following analysis using repeated measures
ANOVAs, significant effects were further characterized by comparing specific treatment conditions using Tukey's HSD test. Prior to analysis, data were assessed for normality and outliers. Circling data were found to be normally distributed using the Shapiro-Wilk test for normality. In checking for outliers, a data point was considered to be an outlier if it fell more than three interquartile ranges (IQR) above or below the median. Using this criterion, 2 points across two rats were identified as outliers in the circling data. These points were fenced to the outer limit (median $\pm 3 \mathrm{IQR}$ ), as there was no reason to believe that the data for these subjects was incorrect. For all experiments, the criterion of significance was set at $p<0.05\left(^{*}\right), p<0.01\left(^{* *}\right)$, and $p<0.001\left(^{* * *}\right)$. 
a

BD 1063
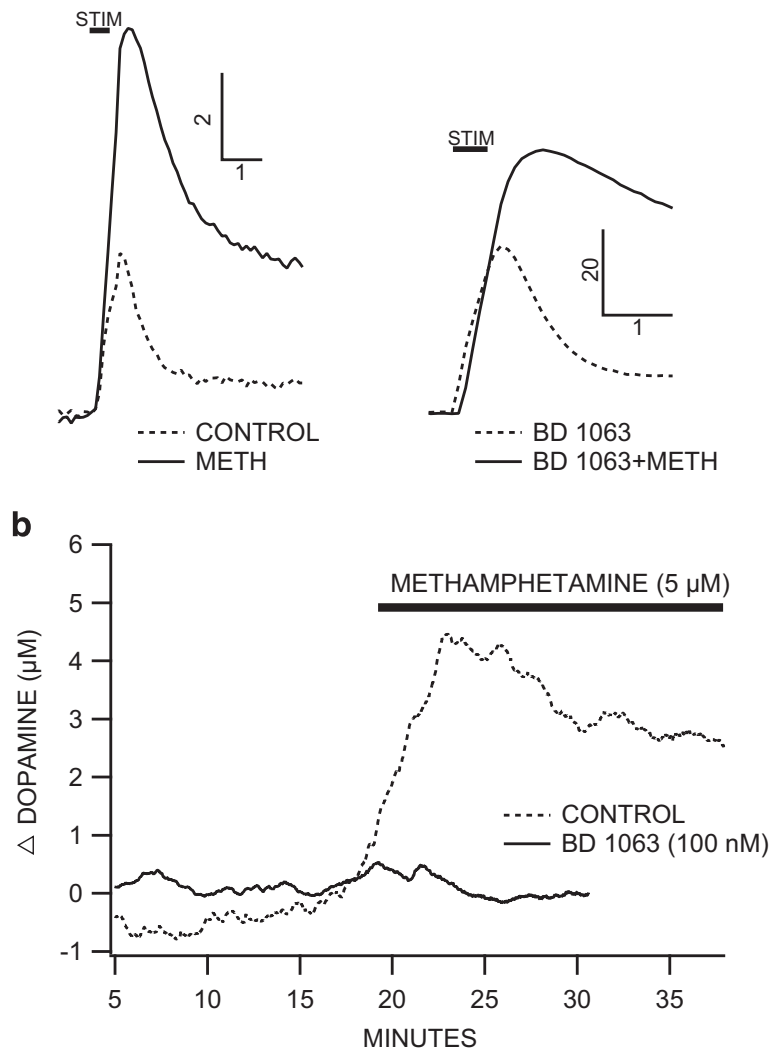

C

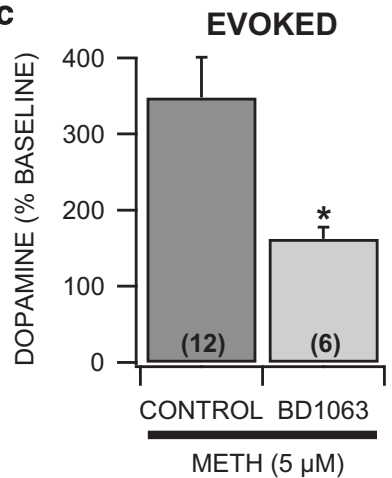

d TEMPOL
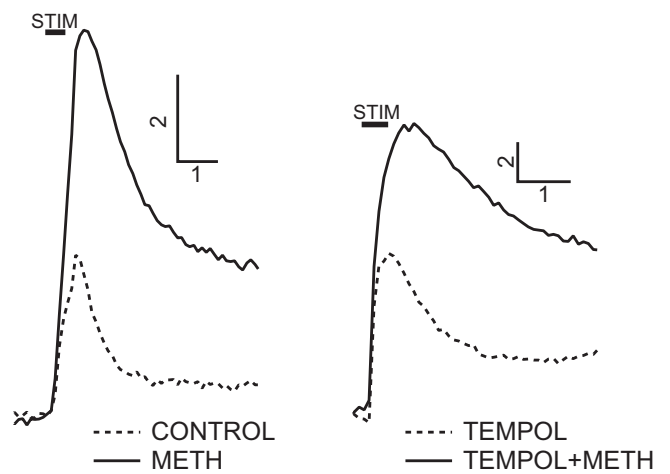

e

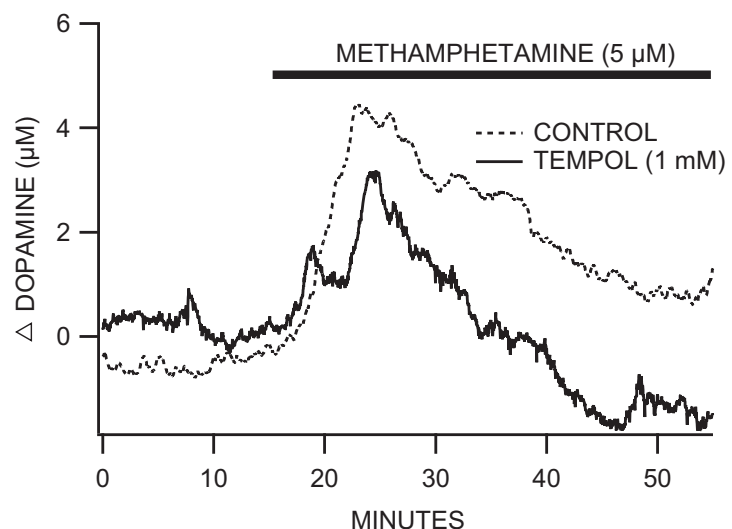

f

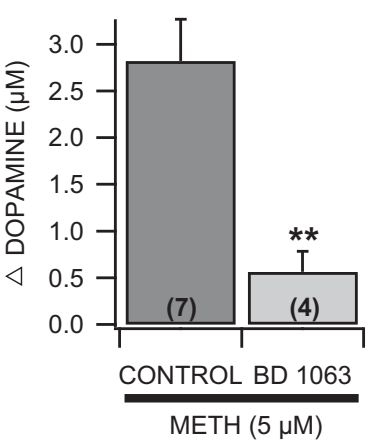

EVOKED

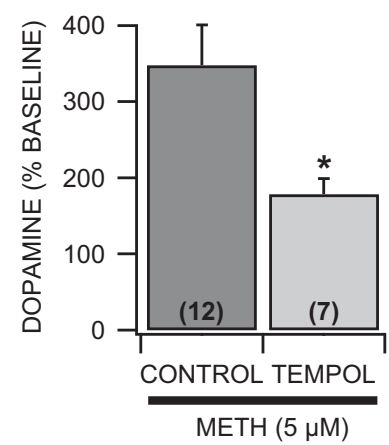

EFFLUX

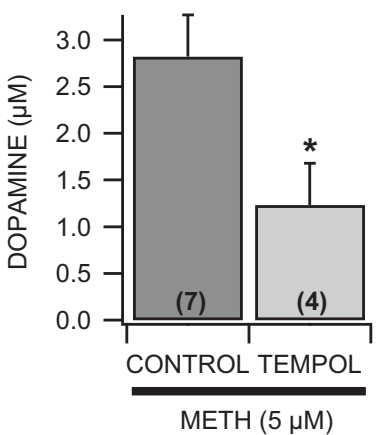

Figure 3 The sigma receptor antagonist BD-1063 reduces methamphetamine-induced dopamine release in the nucleus accumbens core ex vivo. (a) Representative superimposed current vs time plots comparing METH effects on evoked DA release in the NAc core under control conditions vs in the presence of the $\sigma R$ antagonist BD- 1063 (I MM), which moderately reduced METH-induced evoked DA release. (b) Methamphetamine-induced DA efflux was abolished by BD-I063 (I00 nM). (c) BD-I063 significantly reduced both METH-induced evoked DA release (left) and DA efflux (right). Values in parentheses indicate $n$ values. ( $d, e, f)$ TEMPOL, a superoxide dismutase mimetic compound, significantly reduced both METH-induced evoked DA release ( $f$, left) and DA efflux (f, right). Asterisks (*, ${ }^{*} *$ ) represent significance levels $p<0.05$ and $p<0.0$ I, respectively. DA, dopamine; METH, Methamphetamine.

\section{Supplementary Materials and Methods}

For details relating to CFE construction, mass spectrometry and the VMAT2 activity assay methods, please see Supplementary Materials and Methods.

\section{RESULTS}

\section{METH Induces DA Efflux in the NAc Core Independent of Action Potentials or Artificial Stimulation}

Voltammetry was used to evaluate the effects of METH (0.1$100 \mu \mathrm{M})$ on electrically evoked DA release in the NAc core.
METH increased the peak amplitude of the evoked $(20 \mathrm{~Hz}$, 10 pulse) DA signal with an $\mathrm{EC}_{50}$ of $\sim 5 \mu \mathrm{M}$ (Figure $1 \mathrm{a}-\mathrm{c}$; $\mathrm{F}_{4,30}=4.27, p<0.01$ ) and induced spontaneous (i.e., no artificial stimulation) DA efflux with a similar $\mathrm{EC}_{50}$ (Figure 1f; $\mathrm{F}_{4,16}=3.54, p<0.05$ ). The representative trace and voltammograms for non-stimulated DA release were obtained using $100 \mu \mathrm{M}$ METH (Figure 1d and e). Lidocaine $(100 \mu \mathrm{M})$, a voltage-gated sodium channel blocker, reduced electrically stimulated DA release (Figure $2 \mathrm{a}$ and $c$, left; $100 \mu \mathrm{M}$ lidocaine: $\mathrm{F}_{1,16}=15.04, p<0.001$ ), while having no impact on non-stimulated DA release by METH (Figure $2 \mathrm{~b}$ and $c$, right; $100 \mu \mathrm{M}$ lidocaine: $\mathrm{F}_{1,10}=0$, ns). Electrically 
evoked DA concentrations are expressed as a percent change, but averaged raw values were: control $=1.28 \pm 0.52 \mu \mathrm{M}$, Lidocaine $=0.20 \pm 0.05 \mu \mathrm{M}$. The DAT inhibitor GBR-12909 failed to prevent $\mathrm{METH}$-induced increases in stimulated DA release (Figure 2d and f, left; $1 \mu \mathrm{M}$ GBR-12909: $\mathrm{F}_{1,16}=1.94$, ns), but markedly reduced METH-induced non-stimulated DA release (Figure $2 \mathrm{e}$ and $\mathrm{f}$, right; $300 \mathrm{nM}$ GBR-12909: $\left.\mathrm{F}_{1,9}=13.16, p<0.01\right)$, suggesting that nonstimulated release represents DAT-mediated efflux. Averaged raw electrically evoked DA concentrations were: control baseline $=0.57 \pm 0.15 \mu \mathrm{M}$; control $\mathrm{METH}=1.52 \pm 0.32 \mu \mathrm{M}$; experimental baseline $=0.84 \pm 0.27 \mu \mathrm{M}$; GBR-12909 $+\mathrm{METH}=1.48 \pm 0.32 \mu \mathrm{M}$. Therefore, METH enhances two different forms of DA release: action potential-dependent and DAT-mediated efflux.

\section{Sigma Receptors are Involved in METH-Induced Enhancement of DA Release}

With a $K_{\mathrm{i}}$ of $2 \mu \mathrm{M}, \mathrm{METH}$ is a potent agonist of $\sigma 1 \mathrm{Rs}$ (Itzhak, 1993; Nguyen et al, 2005). Therefore, $\sigma 1 \mathrm{R}$ interactions with METH effects on DA release were examined. The $\sigma 1 \mathrm{R}$ selective antagonist BD-1063 (100 nM) had no apparent direct effects on stimulated DA release or uptake (data not shown). BD-1063 prevented METH $(5 \mu \mathrm{M})$ enhancement of stimulated DA release by $\sim 50 \%$ (Figure $3 \mathrm{a}$ and $\mathrm{c}$, left; $100 \mathrm{nM}$ BD-1063: $\left.\mathrm{F}_{1,16}=4.96, p<0.05\right)$ and METH-driven DA efflux by $80 \%$ (Figure $3 \mathrm{~b}$ and $\mathrm{c}$, right; $100 \mathrm{nM}$ BD-1063: $\left.\mathrm{F}_{1,9}=12.97, p<0.01\right)$. These effects appeared to be due to inactivation of $\sigma 1 \mathrm{R}$ and not $\sigma 2 \mathrm{R}$ due to the high affinity of BD-1063 for the $\sigma 1 \mathrm{R}\left(K_{\mathrm{i}}=9 \mathrm{nM}\right.$ for $\sigma 1 \mathrm{R} v s 449 \mathrm{nM}$ for $\sigma 2 \mathrm{R})$. Therefore, $\sigma 1 \mathrm{R}$ activation appears to be involved in METH's effects on both stimulated and non-stimulated DA release.

\section{Antioxidants Prevent METH-Induced DA Release}

METH may influence DA release through its oxidative stress effects via ROS formation (Imam et al, 2001a; Solhi et al, 2014; Walker et al, 2014). Indeed, $\sigma 1 \mathrm{R}$ activation induces ROS formation, which may contribute to METH's effects on DA release. Therefore, the effects of TEMPOL, a superoxide scavenger, on METH $(5 \mu \mathrm{M})$ induced changes in DA release were examined. Similar to previous results (Jang et al, 2017), TEMPOL (1 mM) had no apparent direct effects on stimulated DA release or uptake (data not shown). TEMPOL attenuated METH-induced enhancement of stimulated DA release by $45 \%$ (Figure 3d and f, left; $1 \mathrm{mM}$ TEMPOL: $\mathrm{F}_{1,17}=5.51, p<0.05$ ), and decreased METH-evoked DA efflux by $67 \%$ (Figure $3 e$ and $\mathrm{f}$, right; $1 \mathrm{mM}$ TEMPOL: $\left.\mathrm{F}_{1,10}=5.34, p<0.05\right)$. Averaged raw DA concentrations for TEMPOL are: control baseline $=$ $0.57 \pm 0.15 \mu \mathrm{M}$; control $\mathrm{METH}=1.52 \pm 0.32 \mu \mathrm{M}$; experimental baseline $=0.53 \pm 0.08 \mu \mathrm{M} ; \quad$ TEMPOL $+\mathrm{METH}=0.99 \pm 0.10 \mu \mathrm{M}$. Therefore, ROS production is involved in METH's effects on stimulated DA release and DA efflux.

\section{ROS Impair VMAT2 Function}

ROS formation can lead to protein S-glutathionylation. This post-translational modification involves the covalent conjugation of the thiol group of reduced glutathione (GSH) to cysteine residues on proteins, which then can alter protein function (for review see (Womersley and Uys, 2016)). As METH increases ROS formation, METH may affect VMAT2 function and DA vesicular packaging through this protein modification (Uys et al, 2014). Therefore, the effects of ROS on VMAT2 function and $S$-glutathionylation were examined. As METH has direct effects on VMAT2 function (Fleckenstein et al, 2009), the effects of disulfiram (a molecule that induces ROS formation; $10 \mu \mathrm{M}$ ) and $\mathrm{GSH}$ $(10 \mathrm{mM})$ on VMAT2 function were tested. Disulfiram markedly decreased VMAT2 function in a VMAT2 activity assay using protein purified from fresh mouse NAc tissue (Supplementary Figure $1 \mathrm{~A} ; \mathrm{F}_{1,6}=576.96, p<0.001$ ). Recombinant human VMAT2 protein was exposed to disulfiram and GSH and subsequently analyzed using mass spectrometry. Cys residue 488 was identified as a site susceptible to $S$-glutathionylation (Supplementary Figure 1B). Therefore, ROS formation can induce $S$-glutathionylation of VMAT2 that decreases VMAT2 activity, resulting in impaired vesicular packaging of DA.

\section{Acute METH Causes S-Glutathionylation of VMAT2}

As VMAT2 function was inhibited in vitro, and appeared to involve S-glutathionylation of Cys 488, the effects of METH on VMAT2 Cys 488 S-glutathionylation were tested in vivo. A co-immunoprecipitation assay was used to determine the extent of VMAT2 S-glutathionylation. METH (intraperitoneal, $10 \mathrm{mg} / \mathrm{kg}$ ) increased modified VMAT2 levels. Furthermore, preinjection of BD-1063 (intraperitoneal, $30 \mathrm{mg} / \mathrm{kg}$ ) attenuated METH's effects on VMAT2 S-glutathionylation (Figure 4; $\mathrm{F}_{2,9}=4.26, p=0.006$ ). Therefore, METH-induced $S$-glutathionylation of VMAT2, and subsequent impaired VMAT2 activity involves METH's effects on $\sigma 1 \mathrm{R}$ activity.

\section{METH-Induced Circling Behavior is Dependent on $\sigma 1 \mathrm{R}$}

Amphetamines increase locomotor activity by increasing DA transmission, and co-administration of DA receptor antagonists in the NAc induce circling behavior (Ikeda et al, 2007). Therefore, changes in circling behavior may indicate relative strength of METH's effects on DA release. The involvement of $\sigma 1 \mathrm{R}$ activation in METH's (intraperitoneal, $5 \mathrm{mg} / \mathrm{kg}$ )

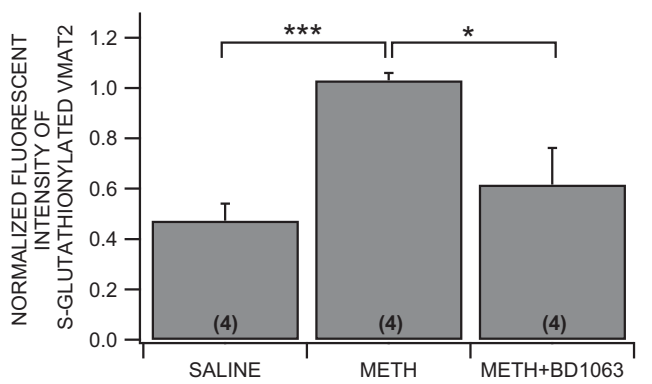

Figure 4 S-glutathionylation of VMAT2 after acute methamphetamine. Fluorescent intensities for S-glutathionylated VMAT2 normalized against total VMAT2 intensity $(n=4)$. Animals injected with METH $(10 \mathrm{mg} / \mathrm{kg}$, IP) show increased levels of S-glutathionylation as compared with the controls. BD- $063(30 \mathrm{mg} / \mathrm{kg}, \mathrm{IP})$ reduces METH-enhancement of S-glutathionylation. Asterisks (*, ****) represent significance levels $p<0.05$ and $p<0.00 \mathrm{I}$, respectively. Values in parentheses indicate $n$ values. METH, Methamphetamine. 
a

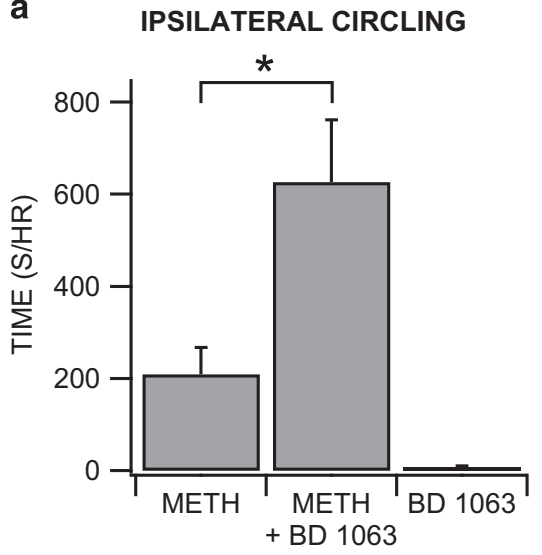

b

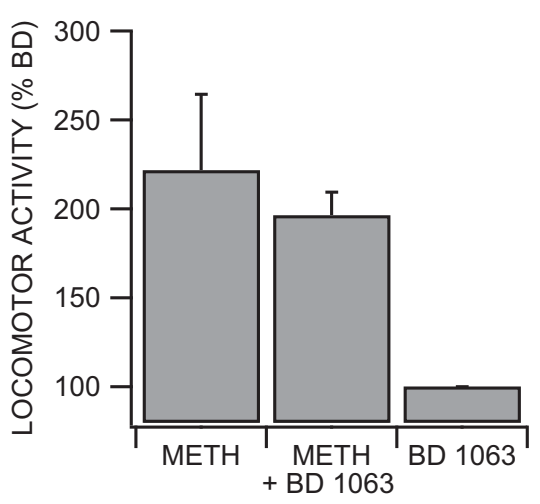

C

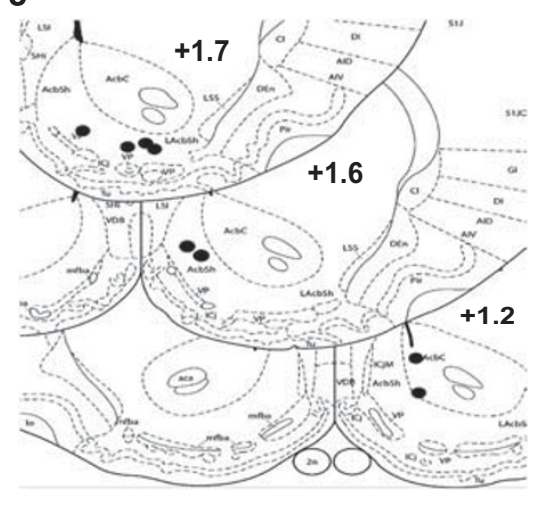

Figure 5 Circling behavior following acute methamphetamine. (a) Intraperitoneal METH induces some, albeit insignificant, circling ipsilateral to a control ACSF infusion through a unilateral guide cannula. When BD- 1063 is dissolved in the ACSF cannula infusion, a systemic METH injection produces profound ipsilateral circling. Unilateral BD-I063 infusion without METH injection fails to induce ipsilateral circling on its own. (b) Systemic METH appears to induce increased locomotor activity. This increase does not appear to be attenuated by cannula infusion of BD-1063 dissolved in ACSF. (c) Anatomical charts showing location of guide cannulas. Asterisk (*) represents significance level $p<0.05$. ACSF, artificial cerebrospinal fluid; METH, Methamphetamine.

effects on circling behavior was tested (Figure 5). One-way ANOVA revealed a main effect of treatment on circling behavior (Figure 5a; $F_{2,}, 12=12.92, p=0.004$ ). Intra-NAc injections of BD-1063 (5 $\mu \mathrm{g})$ increased time spent circling ipsilateral to the hemisphere of the microinjection (METH $+\mathrm{BD})$ compared to ACSF (METH+BD vs METH+ACSF; $t=-4.82, p=0.001, n=8)$ or saline (intraperitoneal) and $\mathrm{BD}-1063$ controls (METH+BD vs saline+BD; $t=3.60$, $p=0.01, n=8$, and $n=6$ respectively). Ipsilateral circling did not differ significantly between METH+ACSF and saline+BD treatments $(t=-1.61, \quad p=0.281)$. Treatment did not appear to affect time spent circling contralateral to the hemisphere of the microinjection (saline+BD: $7.89 \pm 5.20$ vs METH+BD: $377.45 \pm 199.18$ vs METH+ACSF: $\left.121.44 \pm 68.98 ; F_{2,12}=3.01, p=0.1293\right)$. There was a trend toward general increased locomotor activity in the treatment conditions involving METH injections; however, ANOVA did not reveal a significant effect of treatment on locomotor activity (Figure 5b; saline+BD: $100 \pm 0 \%$, METH+BD: $196.51 \pm 12.85 \%, \quad$ METH+ACSF: $221.76 \pm 42.56 \% ; \quad F_{2,8}=$ 6.42, $p=0.0553 ; n=5$ each). Posthumous identification of microinjection location verified that BD-1063 and ACSF microinjections were located in the NAc core and shell regions (Figure 5c). Therefore, inactivation of $\sigma 1 \mathrm{Rs}$ by BD-1063 results in METH-induced circling behavior, likely through reduced METH effects on DA release in BD-1063 ipsilateral infused regions.

\section{DISCUSSION}

Blocking voltage-gated sodium channels prevents action potential-dependent DA release, whereas METH-induced DA efflux was prevented by DAT blockade via GBR-12909, indicating that the mechanisms underlying METH's effects on these two forms of release are fundamentally different (Jones et al, 1998b; Schmitz et al, 2001; Sulzer, 2011). METH has many effects that can increase DA release. For example, amphetamines increase neuronal excitability through increases in DAT-mediated currents, resulting in membrane depolarization and increased firing (Ingram et al, 2002). Amphetamines also increase DA signals at terminals by decreasing DAT activity so that DA signals from multiplepulse stimulations are more sensitive to uptake inhibition (Siciliano et al, 2014). The present study indicates that METH's effects on DA release are more complex than direct interactions with the DAT and VMAT2, involving activation of $\sigma 1$ Rs and subsequent modifications in VMAT2 function. Blocking $\sigma 1$ Rs with BD-1063 attenuated both METHinduced increases in action potential-dependent release and DAT-mediated efflux. METH is a potent $\sigma 1 \mathrm{R}$ agonist (Maurice and $\mathrm{Su}, 2009$ ) and activation of $\sigma 1 \mathrm{R}$ induces ROS production, which then modifies VMAT2 activity through $S$ glutathionylation of Cys 488 .

Previous studies using cumulative concentrations across several hours of METH have shown only decreases in evoked DA release at higher concentrations (John and Jones, 2007; Siciliano et al, 2014), likely due to vesicular depletion effects for these longer experiments. Therefore, to study the DAenhancing effects of METH on evoked release across multiple concentrations, single concentrations were applied to individual slices.

\section{Role of the Sigma Receptor in METH-Induced DA Increases}

The $\sigma 1 \mathrm{R}$ is fundamentally different from classic ligandactivated receptors such as G-protein-coupled receptors and ion channels, in that it is not a plasma membrane receptor but acts primarily as a chaperone receptor (Matsumoto et al, 2014). METH's DA-enhancing effects appear to involve the $\sigma 1 \mathrm{R}$ signaling cascade as the selective $\sigma 1 \mathrm{R}$ antagonist BD-1063 attenuated METH-induced increases in stimulated and non-stimulated release. Cocaine is another $\sigma \mathrm{R}$ agonist (Maurice and Su, 2009), suggesting that similar to METH, cocaine may enhance DA release in part through $\sigma \mathrm{R}$ activation. Indeed, both METH and cocaine increase electrically stimulated DA release (Jang et al, 2015; 2017). However, similar to GBR-12909, cocaine is also a DAT blocker and is likely to prevent any $\sigma 1 \mathrm{R}$-dependent DATmediated DA efflux. Importantly, as a substrate for the DAT, it is possible that METH is more pharmacologically active at intracellular sigma receptors than cocaine, and 
whether cocaine interacts with sigma receptors to influence DA release needs further examination.

\section{METH-Induced Production of ROS}

METH induces oxidative stress in neural tissue (Jang et al, 2015), and recovering METH addicts evince higher levels of oxidative stress (Huang et al, 2013). It is widely assumed that most of the oxidative stress associated with METH abuse results from high levels of extra-vesicular DA that is then oxidized and metabolized (e.g., a byproduct of monoamine oxidase is hydrogen peroxide). However, this does not appears to be the sole source of oxidative stress in DA terminals following METH. For instance, METH also inhibits complexes I, II, III, and IV in the electron transport chain in the mitochondria (for complete review see (Barbosa et al, 2015)), indicating that there are other potential sources of oxidative stress. Presently, TEMPOL was used to reduce superoxide exposure caused by METH. As TEMPOL attenuated METH-induced increases in DA release, it is likely that METH-induced oxidative stress is upstream to DA vesicular leakage and subsequent DA auto-oxidation. Moreover, TEMPOL attenuates mitochondrial-induced oxidative damage by METH and METH-induced sensitization (Shiba et al, 2011), as well as with cocaine in vivo (Jang et al, 2015), indicating that METH's and cocaine's oxidative stress effects are involved in aspects of psychostimulant reinforcement.

\section{METH-Induced Oxidative Stress and VMAT2}

The mechanisms involved in METH-triggered oxidative stress are still being elucidated. Chronic oxidative stress from METH exposure is thought to contribute to its neurotoxic effects (Yu et al, 2015), but the immediate consequences of ROS are not well understood. Reactive radicals can lead to $S$ glutathionylation, a post-translational modification on cysteine residues that could lead to functional changes in target proteins (Uys et al, 2014). Presently, high-resolution mass spectrometry experiments revealed that disulfiram induced S-glutathionylation (Rossi et al, 2006) on Cys 488 of VMAT2. Disulfiram decreased VMAT2 function, presumably through this Cys modification. Furthermore, a single in vivo METH exposure increased VMAT2 Cys 488 S-glutathionylation. Decreased VMAT function is thought to prevent vesicles from maintaining 'normal' DA concentrations, resulting in DA leakage (Sulzer, 2011), either through inherently leaky vesicles (Floor et al, 1990) or through a disrupted proton gradient (Freyberg et al, 2016). Therefore, METH-induced decreases in VMAT function may result in increased cytosolic DA. Increased cytosolic DA could then overwhelm the active transport of the DAT, leading to DA efflux through the DAT. Concurrent to these effects, build-up and breakdown of DA increases oxidative stress (both inside and outside of the cell), as DA metabolites include superoxide, hydrogen peroxide, and 3,4-dihydroxyphenylacetaldehyde (DOPAL) (Casida et al, 2014; Kita et al, 1999; LaVoie and Hastings, 1999). This adds to METH-induced neurotoxicity. Supporting these findings, it has been shown that increasing expression of VMAT2 protects against METH neurotoxicity (Lohr et al, 2015) and that chronic METH users show depleted VMAT2 expression (Johanson et al, 2006).
In the present study, METH enhanced evoked DA release, which TEMPOL application attenuated. This suggests that similar to METH-induced DA efflux, increases in evoked DA release also involve the formation of ROS. However, the mechanisms behind ROS-induced increases in DA release are unclear and may involve multiple effects on DA terminals. Furthermore, it is questionable that this effect involves VMAT2 impairment and resultant vesicular depletion, as the VMAT2 inhibitor reserpine only appears to decrease evoked DA release (Yorgason et al, 2017; Gantz et al, 2013). METH-induced ROS formation includes nonenzymatic formation of nitric oxide (Friend et al, 2014). Nitric oxide has been shown to increase evoked DA release through direct and indirect effects on DA terminals, an effect that is prevented by nitric oxide scavengers (Hartung et al, 2011). Therefore, METH likely increases DA release in part through nitric oxide formation and downstream effects on local interneuron activity onto DA terminals. TEMPOL is a nitric oxide scavenger and therefore should attenuate any nitric oxide-induced increases in DA release. Importantly, the multiple-pulse stimulations used in the present study may be particularly sensitive to METH-induced increases due to increased competition at the DAT due to additional DA (Siciliano et al, 2014). Indeed, METH increases evoked DA release more readily by multiple $v s$ single-pulse stimulations (Siciliano et al, 2014; John and Jones, 2007). However, nitric oxide enhanced DA release is also greater during multiple-pulse stimulations (Hartung et al, 2011). Therefore, METH-induced increases in evoked DA release appear to involve multiple factors.

\section{METH-Induced Circling Behavior}

METH treatment following 6-OHDA lesions of the mesolimbic pathway has been shown to produce ipsilateral circling behavior in rats (Ali et al, 1995; Jalewa et al, 2017). In addition, unilateral treatment with $\mathrm{D} 1 / \mathrm{D} 2$ receptor agonists can induce contralateral circling, an effect that can be abolished by ipsilateral injection of a D1/D2 receptor antagonist (Ikeda et al, 2007; Saigusa et al, 1993). This indicates that circling results from an imbalance in DA transmission between the two hemispheres. In this study, unilateral injections of BD-1063 in the ventral striatum produced an increase in ipsilateral circling behavior following treatment with systemic METH. As BD-1063 decreases METH-induced DA increases ex vivo, it is likely producing an imbalance in vivo in NAc DA transmission to produce ipsilateral circling behavior. Thus, $\sigma 1 \mathrm{R}$ activation appears to have a key role in METH's ability to increase DA signaling and related behaviors. As amphetamines enhance conditioned-learning and goal-oriented behavior through increased DA release during phasic DA firing (Knutson et al, 2004; Wyvell and Berridge, 2000), it is likely that drugs targeting $\sigma 1 \mathrm{R}$ may help reduce the reinforcing valence of METH, though this will need to be tested explicitly.

\section{CONCLUSIONS}

On the basis of the present studies, a model of METH's action on $\sigma 1 \mathrm{R}$ and downstream effects in DA terminals is proposed (Supplementary Figure 2). METH enters the DA 
terminal through the DAT, attenuating DA clearance to increase DA release. Once inside the cell, METH activates the $\sigma 1 \mathrm{R}$, increasing $\mathrm{Ca}^{2+}$ signaling between the smooth ER and the mitochondria (Ruscher and Wieloch, 2015). This release of intracellular $\mathrm{Ca}^{2+}$ increases evoked DA release (Fernandes et al, 2004; Kataoka et al, 1994) through undescribed mechanisms. Increased intracellular $\mathrm{Ca}^{2+}$ then disrupts mitochondrial function and results in superoxide production. Superoxide (mechanistically related to many other ROS (Bellinger et al, 2009)) triggers S-glutathionylation of VMAT2, which impairs VMAT2 function and allows for DA-containing vesicles to be depleted. DA released from vesicle pools flows out through the compromised DAT. Altogether, the present data indicate that the $\sigma 1 \mathrm{R}$ and related oxidative stress machinery may be appropriate targets for treatment and prevention of METH abuse and dependence.

\section{FUNDING AND DISCLOSURE}

This work was supported by NIH Grants AA020919 and DA035958 to SCS, DA040409 to JTY, and AA024426 and GM103542-02 to JDU. The authors declare no conflict of interest.

\section{ACKNOWLEDGMENTS}

We would like to thank the personnel who contributed to this project, namely Dr. Jacqueline Womersley, Dr. Bryan Blummel, Andrew Perez, Christopher Schow, Gilbert Marchant, Spencer McCarthy, and Mark Woodbury. We would like to dedicate this work in memory of Samuel I. Shin.

\section{REFERENCES}

Ali SF, Kordsmeier KJ, Gough B (1995). Drug-induced circling preference in rats. Correlation with monoamine levels. Mol Neurobiol 11: 145-154.

Barbosa DJ, Capela JP, Feio-Azevedo R, Teixeira-Gomes A, Bastos Mde L, Carvalho F (2015). Mitochondria: key players in the neurotoxic effects of amphetamines. Arch Toxicol 89: 1695-1725.

Bellinger FP, Raman AV, Reeves MA, Berry MJ (2009). Regulation and function of selenoproteins in human disease. Biochem J 422: $11-22$.

Casida JE, Ford B, Jinsmaa Y, Sullivan P, Cooney A, Goldstein DS (2014). Benomyl, aldehyde dehydrogenase, DOPAL, and the catecholaldehyde hypothesis for the pathogenesis of Parkinson's disease. Chem Res Toxicol 27: 1359-1361.

Chu PW, Seferian KS, Birdsall E, Truong JG, Riordan JA, Metcalf CS et al (2008). Differential regional effects of methamphetamine on dopamine transport. Eur J Pharmacol 590: 105-110.

Eshleman AJ, Henningsen RA, Neve KA, Janowsky A (1994). Release of dopamine via the human transporter. Molecular Pharmacology 45: 312-316.

Fernandes VM, Romano-Silva MA, Gomes DA, Prado MA, Santos TM, Gomez MV (2004). Dopamine release evoked by beta scorpion toxin, tityus gamma, in prefrontal cortical slices is mediated by intracellular calcium stores. Cell Mol Neurobiol 24: 757-767.

Fleckenstein AE, Volz TJ, Hanson GR (2009). Psychostimulantinduced alterations in vesicular monoamine transporter- 2 function: neurotoxic and therapeutic implications. Neuropharmacology 56(Suppl 1): 133-138.
Floor E, Leventhal PS, Schaeffer SF (1990). Partial purification and characterization of the vacuolar $\mathrm{H}(+)$-ATPase of mammalian synaptic vesicles. J Neurochem 55: 1663-1670.

Friend DM, Fricks-Gleason AN, Keefe KA (2014). Is there a role for nitric oxide in methamphetamine-induced dopamine terminal degeneration? Neurotox Res 25: 153-160.

Freyberg Z, Sonders MS, Aguilar JI, Hiranita T, Karam CS, Flores J et al (2016). Mechanisms of amphetamine action illuminated through optical monitoring of dopamine synaptic vesicles in Drosophila brain. Nat Commun 7: 10652.

Fukunaga K, Shinoda Y, Tagashira H (2015). The role of SIGMAR1 gene mutation and mitochondrial dysfunction in amyotrophic lateral sclerosis. J Pharmacol Sci 127: 36-41.

Gantz SC, Bunzow JR, Williams JT (2013). Spontaneous inhibitory synaptic currents mediated by a g protein-coupled receptor. Neuron 78: 807-812.

Han DD, Gu HH (2006). Comparison of the monoamine transporters from human and mouse in their sensitivities to psychostimulant drugs. BMC Pharmacol 6: 6.

Hartung H, Threlfell S, Cragg SJ (2011). Nitric oxide donors enhance the frequency dependence of dopamine release in nucleus accumbens. Neuropsychopharmacology 36: 1811-1822.

Hiranita T, Soto PL, Tanda G, Kopajtic TA, Katz JL (2013). Stimulants as specific inducers of dopamine-independent sigma agonist self-administration in rats. J Pharmacol Exp Ther 347: $20-29$.

Huang MC, Lin SK, Chen CH, Pan CH, Lee CH, Liu HC (2013). Oxidative stress status in recently abstinent methamphetamine abusers. Psychiatry Clin Neurosci 67: 92-100.

Ikeda H, Kotani A, Koshikawa N, Cools AR (2007). A vehicle injection into the right core of the nucleus accumbens both reverses the region-specificity and alters the type of contralateral turning elicited by unilateral stimulation of dopamine D2/D3 and D1 receptors in the left core of the nucleus accumbens. Eur $J$ Pharmacol 577: 64-70.

Imam SZ, el Yazal J, Newport GD, Itzhak Y, Cadet JL, Slikker W Jr. et al (2001a). Methamphetamine-induced dopaminergic neurotoxicity: role of peroxynitrite and neuroprotective role of antioxidants and peroxynitrite decomposition catalysts. Ann $N$ $Y$ Acad Sci 939: 366-380.

Imam SZ, Newport GD, Itzhak Y, Cadet JL, Islam F, Slikker W Jr. et al (2001b). Peroxynitrite plays a role in methamphetamineinduced dopaminergic neurotoxicity: evidence from mice lacking neuronal nitric oxide synthase gene or overexpressing copper-zinc superoxide dismutase. J Neurochem 76: 745-749.

Ingram SL, Prasad BM, Amara SG (2002). Dopamine transportermediated conductances increase excitability of midbrain dopamine neurons. Nat Neurosci 5: 971-978.

Itzhak Y (1993). Repeated methamphetamine-treatment alters brain sigma receptors. Eur J Pharmacol 230: 243-244.

Jalewa J, Sharma MK, Gengler S, Holscher C (2017). A novel GLP-1/ GIP dual receptor agonist protects from 6-OHDA lesion in a rat model of Parkinson's disease. Neuropharmacology 117: 238-248.

Jang EY, Ryu YH, Lee BH, Chang SC, Yeo MJ, Kim SH et al (2015). Involvement of reactive oxygen species in cocaine-taking behaviors in rats. Addict Biol 20: 663-675.

Jang EY, Yang CH, Hedges DM, Kim SP, Lee JY, Ekins TG et al (2017). The role of reactive oxygen species in methamphetamine self-administration and dopamine release in the nucleus accumbens. Addict Biol. 22: 1304-1315.

Johanson CE, Frey KA, Lundahl LH, Keenan P, Lockhart N, Roll J et al (2006). Cognitive function and nigrostriatal markers in abstinent methamphetamine abusers. Psychopharmacology 185: 327-338.

John CE, Jones SR (2007). Voltammetric characterization of the effect of monoamine uptake inhibitors and releasers on dopamine and serotonin uptake in mouse caudate-putamen and substantia nigra slices. Neuropharmacology 52: 1596-1605. 
Jones SR, Gainetdinov RR, Jaber M, Giros B, Wightman RM, Caron MG (1998a). Profound neuronal plasticity in response to inactivation of the dopamine transporter. Proc Natl Acad Sci USA 95: 4029-4034.

Jones SR, Gainetdinov RR, Wightman RM, Caron MG (1998b). Mechanisms of amphetamine action revealed in mice lacking the dopamine transporter. J Neurosci 18: 1979-1986.

Kataoka Y, Koizumi S, Niwa M, Shibaguchi H, Shigematsu K, Kudo $Y$ et al (1994). Endothelin-3 stimulates inositol 1,4,5-trisphosphate production and $\mathrm{Ca} 2+$ influx to produce biphasic dopamine release from rat striatal slices. Cell Mol Neurobiol 14: 271-280.

Katz JL, Su TP, Hiranita T, Hayashi T, Tanda G, Kopajtic T et al (2011)A role for sigma receptors in stimulant self administration and addictionPharmaceuticals Basel 4: 880-914.

Kaushal N, Elliott M, Robson MJ, Iyer AK, Rojanasakul Y, Coop A et al (2012). AC927, a sigma receptor ligand, blocks methamphetamine-induced release of dopamine and generation of reactive oxygen species in NG108-15 cells. Mol Pharmacol 81: 299-308.

Kita T, Takahashi M, Kubo K, Wagner GC, Nakashima T (1999). Hydroxyl radical formation following methamphetamine administration to rats. Pharmacol Toxicol 85: 133-137.

Knutson B, Bjork JM, Fong GW, Hommer D, Mattay VS, Weinberger DR (2004). Amphetamine modulates human incentive processing. Neuron 43: 261-269.

LaVoie MJ, Hastings TG (1999). Dopamine quinone formation and protein modification associated with the striatal neurotoxicity of methamphetamine: evidence against a role for extracellular dopamine. J Neurosci 19: 1484-1491.

Lohr KM, Stout KA, Dunn AR, Wang M, Salahpour A, Guillot TS et al (2015). Increased vesicular monoamine transporter 2 (VMAT2; Slc18a2) protects against methamphetamine toxicity. ACS Chem Neurosci 6: 790-799.

Matsumoto RR, Nguyen L, Kaushal N, Robson MJ (2014). Sigma (sigma) receptors as potential therapeutic targets to mitigate psychostimulant effects. Adv Pharmacol 69: 323-386.

Maurice T, Su TP (2009). The pharmacology of sigma-1 receptors. Pharmacol Ther 124: 195-206.

McFadden LM, Vieira-Brock PL, Hanson GR, Fleckenstein AE (2015). Prior methamphetamine self-administration attenuates the dopaminergic deficits caused by a subsequent methamphetamine exposure. Neuropharmacology 93: 146-154.

Nguyen EC, McCracken KA, Liu Y, Pouw B, Matsumoto RR (2005). Involvement of sigma (sigma) receptors in the acute actions of methamphetamine: receptor binding and behavioral studies. Neuropharmacology 49: 638-645.

Nguyen L, Kaushal N, Robson MJ, Matsumoto RR (2014). Sigma receptors as potential therapeutic targets for neuroprotection. Eur J Pharmacol 743: 42-47.

Robinson TE, Berridge KC (1993). The neural basis of drug craving: an incentive-sensitization theory of addiction. Brain Res Brain Res Rev 18: 247-291.

Rossi R, Giustarini D, Dalle-Donne I, Milzani A (2006). Protein S-glutathionylation and platelet anti-aggregating activity of disulfiram. Biochem Pharmacol 72: 608-615.

Rothman RB, Baumann MH (2003). Monoamine transporters and psychostimulant drugs. Eur J Pharmacol 479: 23-40.

Ruscher K, Wieloch T (2015). The involvement of the sigma-1 receptor in neurodegeneration and neurorestoration. J Pharmacol Sci 127: 30-35.
Saigusa T, Koshikawa N, Kitamura M, Kobayashi M (1993). Reevaluation of the two-component hypothesis for turning behaviour by manipulating activities in the striatum and the nucleus accumbens of intact rats. Eur J Pharmacol 237: 161-168.

Schmitz Y, Lee CJ, Schmauss C, Gonon F, Sulzer D (2001). Amphetamine distorts stimulation-dependent dopamine overflow: effects on D2 autoreceptors, transporters, and synaptic vesicle stores. J Neurosci 21: 5916-5924.

Shiba T, Yamato M, Kudo W, Watanabe T, Utsumi H, Yamada K (2011). In vivo imaging of mitochondrial function in methamphetamine-treated rats. Neuroimage 57: 866-872.

Siciliano CA, Calipari ES, Ferris MJ, Jones SR (2014). Biphasic mechanisms of amphetamine action at the dopamine terminal. $J$ Neurosci 34: 5575-5582.

Sitte HH, Huck S, Reither H, Boehm S, Singer EA, Pifl C (1998). Carrier-mediated release, transport rates, and charge transfer induced by amphetamine, tyramine, and dopamine in mammalian cells transfected with the human dopamine transporter. $J$ Neurochem 71: 1289-1297.

Solhi H, Malekirad A, Kazemifar AM, Sharifi F (2014). Oxidative stress and lipid peroxidation in prolonged users of methamphetamine. Drug Metab Lett 7: 79-82.

Steffensen SC, Taylor SR, Horton ML, Barber EN, Lyle LT, Stobbs $\mathrm{SH}$ et al (2008). Cocaine disinhibits dopamine neurons in the ventral tegmental area via use-dependent blockade of GABA neuron voltage-sensitive sodium channels. Eur J Neurosci 28: 2028-2040.

Su TP, Hayashi T, Maurice T, Buch S, Ruoho AE (2010). The sigma1 receptor chaperone as an inter-organelle signaling modulator. Trends Pharmacol Sci 31: 557-566.

Sulzer D (2011). How addictive drugs disrupt presynaptic dopamine neurotransmission. Neuron 69: 628-649.

Sulzer D, Sonders MS, Poulsen NW, Galli A (2005). Mechanisms of neurotransmitter release by amphetamines: a review. Prog Neurobiol 75: 406-433.

Uys JD, Mulholland PJ, Townsend DM (2014). Glutathione and redox signaling in substance abuse. Biomed Pharmacother 68: 799-807.

Walker J, Winhusen T, Storkson JM, Lewis D, Pariza MW, Somoza $\mathrm{E}$ et al (2014). Total antioxidant capacity is significantly lower in cocaine-dependent and methamphetamine-dependent patients relative to normal controls: results from a preliminary study. Hum Psychopharmacol 29: 537-543.

Womersley JS, Uys JD (2016). S-glutathionylation and redox protein signaling in drug addiction. Prog Mol Biol Transl Sci 137: 87-121.

Wyvell CL, Berridge KC (2000). Intra-accumbens amphetamine increases the conditioned incentive salience of sucrose reward: enhancement of reward "wanting" without enhanced "liking" or response reinforcement. J Neurosci 20: 8122-8130.

Yorgason JT, Espana RA, Jones SR (2011). Demon voltammetry and analysis software: analysis of cocaine-induced alterations in dopamine signaling using multiple kinetic measures. J Neurosci Methods 202: 158-164.

Yorgason JT, Zeppenfeld DM, Williams JT (2017). Cholinergic interneurons underlie spontaneous dopamine release in nucleus accumbens. J Neurosci 37: 2086-2096.

Yu S, Zhu L, Shen Q, Bai X, Di X (2015). Recent advances in methamphetamine neurotoxicity mechanisms and its molecular pathophysiology. Behav Neurol 2015: 103969.

Supplementary Information accompanies the paper on the Neuropsychopharmacology website (http://www.nature.com/npp) 